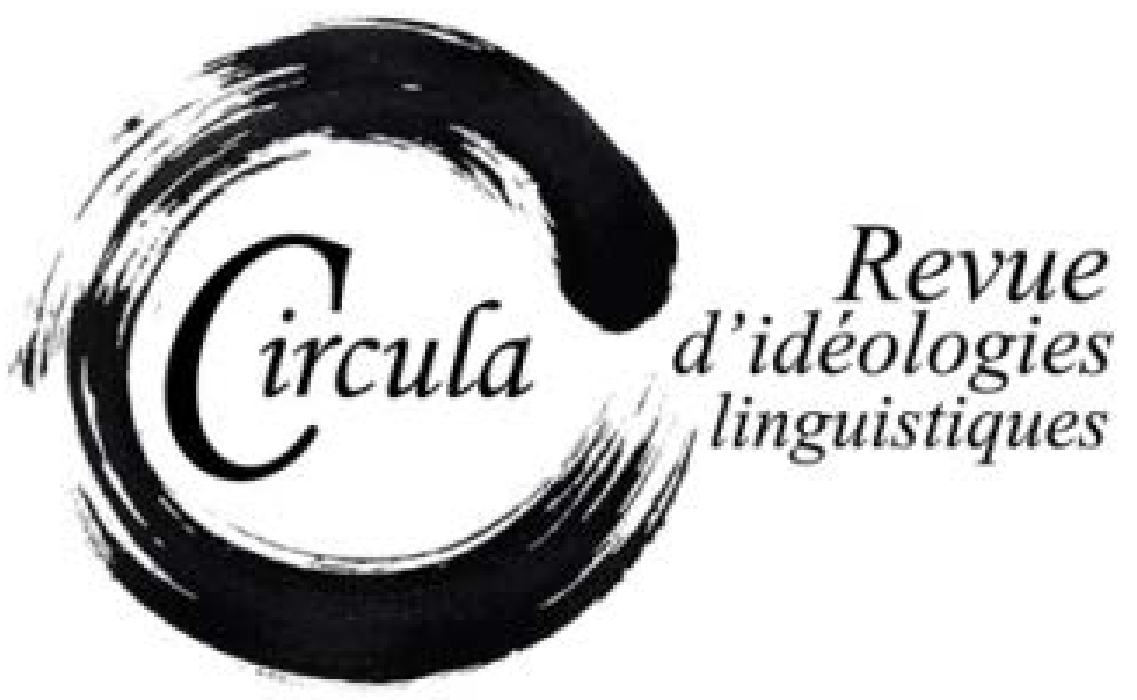

TITRE: QUESTIONI INTORNO ALLA LINGUA NELLE RIVISTE LETTERARIE DEL PRIMO SETTECENTO Auteur(s): Merida, Raphael, UniversitÀ degli StudI DI Messina

Revue: Circula, NumÉro 5

PAgES: $7-23$

ISSN: 2369-6761

DiRecteurs: Wim Remysen, SABIne SCHWARZE ET JUAN ANTONIO EnNiS

URI: HTTP://HDL.HANDLE.NET/11143/11227

DOI: HTTPS://DOI.ORG/10.17118/11143/11227 


\title{
Questioni intorno alla lingua nelle riviste letterarie del primo Settecento
}

Raphael Merida, Università degli Studi di Messina rmerida@unime.it

\begin{abstract}
Riassunto: La ricerca indaga alcuni articoli presenti nei giornali settecenteschi, con particolare attenzione alle riviste letterarie della prima metà del secolo. Sotto le vesti di giornalisti, gli intellettuali dell'epoca proponevano le proprie riflessioni linguistiche attraverso saggi e recensioni di opere lessicografiche, incentivando a un maggior interesse nei confronti dei linguaggi speciali. L'italiano così veniva visto come una lingua da arricchire con lo studio della sua grammatica e l'ampliamento lessicale, necessari alla corretta comunicazione. Scopo del lavoro è centrare l'obiettivo sulla sensibilità linguistica dei questi giornali che, si vedrà, in alcuni casi saranno precursori ideologici dei più importanti fogli periodici del secondo Settecento. Si dimostrerà come l'apertura alla modernità e i tentativi di educazione linguistica facciano da tema comune agli articoli presi in esame.
\end{abstract}

Parole chiave: riviste letterarie; storia della lingua italiana; lingue speciali; questione della lingua.

\begin{abstract}
The research deals with a few articles found in papers from the $18^{\text {th }}$ century, with particular attention given to papers from the first half of the century. Scholars of that time, like modern journalists, would introduce their linguistic thoughts through essays and reviews of lexicographic works, aiming at a deeper interest in specialised languages. Italian has thus been thought of as a language to study in depth through its grammar and lexical growth - both necessary for a correct way of speaking. The aim of this work is to focus on the linguistic sensitivity of these papers that, as you will read, will result in being ideological forerunners of the most important periodicals of the second half of the $18^{\text {th }}$ century. This research will prove how the openness to modernity and the attempts at linguistic education are the themes behind most of the articles.
\end{abstract}

Key words: literary papers; history of the Italian language; specialised languages; question of the language. 


\section{Introduzione}

Nel volume L'italiano in Europa Gianfranco Folena dedicava il primo capitolo al «rinnovamento linguistico del Settecento italiano", attribuendo ai giornali il merito di aver imposto la circolazione della cultura e di aver gettato le fondamenta per una «lingua comune» (Folena, 1983: 7-10). Bisognerebbe soffermarsi sul termine «rinnovamento» che, secondo la percezione degli studiosi ${ }^{1}$, non deve essere identificato «tout court [...] col fenomeno dell'influenza del francese», ed è preferibile alla voce «crisi, termine che mi piacerebbe», scrive Folena, «riservare per alcune manifestazioni particolari ed estreme» (Folena, 1983: 8). Sulla scorta di quanto detto si aggiunge il commento di Luca Serianni che, nel tracciare un quadro della lingua italiana del Settecento, riprende la voce «rinnovamento», oltrepassando quindi l'eredità della critica letteraria che identificava il secolo dei lumi con la crisi linguistica dell'italiano ${ }^{2}$. Nel presente contributo si parlerà perciò di un rinnovamento della lingua e non di un suo progressivo deterioramento: ciò sarà ben visibile nei giornali letterari, senza i quali «non si può pensare alla spinta riformatrice settecentesca e, più specificatamente, all'azione dei 'lumi'» (Matarrese, 1993: 41). Le riviste letterarie, che qui ci interessano, sono contraddistinte da un grado alto di specificità degli argomenti trattati, e quindi non sempre adatti al pubblico. L'interesse per ambiti settoriali vari rende possibile stabilire la sensibilità linguistica presente nei giornali dell'epoca.

Prima di far emergere i principali tratti distintivi, è bene tracciare una linea di confine post e ante quem: per questo, al fine di delineare il quadro della ricerca, si forniranno sondaggi relativi a un corpus di sei riviste letterarie (Galleria di Minerva, Giornale de' letterati d'Italia, L'osservatore veneto, Le Novelle Letterarie, Le Osservazioni letterarie, La Frusta Letteraria) ${ }^{3}$. La scelta dei testi è motivata principalmente dalla presenza di illustri protagonisti nell'ambiente giornalistico di primo Settecento: nel presente articolo si vedrà come diverse volte compaiano gli stessi nomi all'interno di più riviste letterarie (ad es. Zeno che per motivi ideologici si sposta dalla Galleria di Minerva al Giornale de' letterati d'Italia).

1. Alfredo Schiaffini può essere considerato il primo ad aver introdotto il concetto di "crisi" / "rinnovamento" linguistico settecentesco: cf. Schiaffini (1950; 1975).

2. Cf. Serianni (1998: 187); sul termine «rinnovamento» si esprime anche Coletti (2000: 206).

3. Lo spoglio è stato condotto su un campione di venti numeri per rivista. Si adotteranno le seguenti sigle: Galleria, GLI, Osservatore, NL, OL e Frusta (per il Supplemento al Giornale de' letterati d'Italia la sigla sarà GLIsup); dopo ogni citazione, inoltre, si inserirà il nome dell'autore (se presente), il titolo della rivista, l'anno, il volume o il tomo, il numero di pagina. Riporto le annate all'interno delle quali è contenuto un commento linguistico a un articolo o una recensione: Galleria 1696, 1697; GLI 1710, 1711, 1714, 1740; GLIsup 1722; OL 1737; NL 1740, 1747, 1770; Osservatore 1761; Frusta 1763. Ho preferito concentrarmi sui dati provenienti dal commento degli articoli più significativi tra quelli individuati. 


\section{Le aperture al dibattito linguistico della Galleria di Minerva}

Si partirà dal 1696, anno di fondazione della Galleria di Minerva. La premessa ai Letterati d'Europa, a firma del tipografo Girolamo Albrizzi, oltre a manifestare l'apertura di una «publica Galeria alle bone lettere, dove possano queste concorrere, ed essere esposte a gl'occhi del Mondo erudito» (Albrizzi, 1696: II), dichiarava il motivo della scelta del titolo; per cui la Galleria si sarebbe potuta prefigurare come una biblioteca nella quale esporre, cioè presentare, testi editi e inediti, recensioni e lettere, sulla scorta dei giornali europei che già circolavano negli ambienti scientifici. Dai vari carteggi degli intellettuali dell'epoca e dagli studi recenti ${ }^{4}$, è stato fatto notare che all'inizio della sua vita la Galleria poteva vantare la «presenza redazionale di un gruppo colto, tra cui il cosmografo Vincenzo Maria Coronelli, Scipione Maffei, Antonio Vallisneri» (Di Brazzà, 2012: 159) e Apostolo Zeno. Abbiamo pocanzi accennato ai vari interessi scientifici destinati a concretizzarsi in interventi riguardanti l'astronomia, la matematica, l'arte antiquaria; ma ai fini dell'intervento, ci si soffermerà esclusivamente sulle opinioni linguistiche.

Scorrendo gli indici della Galleria, è stato possibile accorgersi di due titoli assai interessanti: (1) Lettera del Sig. Abbate Michel Angelo Fardella Professore di Astronomia e Meteore nello studio di Padova al Signore N.N. in cui per rintracciare colla maggiore facilità il vero metodo di studiare, brevemente s'espongono la corrutione et abusi dele umane scienze, i vitii e difetti de' letterati, seguita da (2) Lettera seconda del Sig. Abbate Michel Angelo Fardella Professore [... in in cui si dimostra quanto sia presentemente corrotta, ed allontanata dal suo primo istituto l'arte di parlare. Decisamente più affine ai problemi di natura linguistica è la seconda lettera, nella quale Fardella scaglia una forte invettiva contro il malcostume moderno, fonte di corruzione e disinteresse della lingua italiana. Procedendo nella lettura della seconda lettera, emerge il binario su cui muove l'idea di Fardella, che identifica due lingue: la materna, chiamata «volgare», e la letterale, cioè latina: «E per ordinare il nostro ragionamento all'uso ed utilità della nostra Italia, io considero due lingue, o siano maniere, e regole di parlare, la materna che volgare appellasi, e la letterale che latina chiamiamo» (Fardella, Galleria, 1697, II: 4).

Vediamo più davvicino alcuni interventi del professore patavino, dai quali emerge una forte carica conservatrice e bembiana, specialmente nei confronti del lessico:

I'Idioma Italiano [...] s'apprende principalmente da' buoni libri, ed in gran parte s'esercita nella corte di Roma, e nelle più floride città della Toscana; nel resto delle altre provincie molto degenera dal suo istituto, e nativa purità, corrotto per la mescolanza di voci straniere e barbare, introdotte dal capriccio del volgo. (Fardella, 1697, Galleria II: 4)

Il passo appena citato (che suggella le infinite considerazioni già avviate dagli intellettuali di fine Cinque e Seicento) interessa soprattutto per la sensibilità linguistica che in quel periodo s'insinuava negli scienziati e che in anni precoci entrava tra le pagine di una rivista letteraria. Per Fardella, dunque,

4. Si veda ad esempio Scotti Morgana 1983. 
la lingua adottata oltre i confini toscani si configurava in un guazzabuglio di voci italiane e straniere che formavano, a suo dire, una «lingua popolare» che s'impara non con le norme dei grammatici, ma con l'uso $0^{5}$. A proposito dei grammatici Fardella, inoltre, intervenendo con l'accusa di troppa riverenza verso la lingua latina, definita ormai «lingua morta» e non più utile ai fini pratici, mostrava un aspetto progressivo del razionalismo linguistico, anticipatore della pedagogia linguistica settecentesca e continuatore dell'idea galileiana sull'uso dell'italiano nelle opere scientifiche, a discapito del latino (cf. Pennisi, 1985: 83-107), che, a sua volta, era considerato «più espressivo, brieve e maestoso della nostra [lingua], e perciò semplicemente necessario per apprendere con maggior frutto e dignità, le liberali dottrine» (Fardella, 1697, Galleria II: 4 [corsivi miei]) e che non permetteva l'esercizio dell'italiano nelle materie scientifiche ${ }^{6}$. Le tesi condotte dal Fardella risuonano di un tono pienamente illuminista e difendono con fermezza l'italiano, il cui studio è ritenuto essenziale e necessario per poter creare un adeguato vocabolario scientifico e per trovare la piena coscienza di sé. Subito dopo la pubblicazione dei primi numeri della Galleria, Albrizzi preferì cambiare strategia editoriale, offrendo largo spazio alle scienze antiquarie: fatto che, come si vedrà, conobbe la disapprovazione di alcuni collaboratori, che preferirono fondare, ad esempio, il Giornale de' letterati d'Italia.

\section{Gli interessi tecnici del Giornale de' letterati d'Italia}

A distanza di quasi un ventennio rimane altissimo l'interesse per i settori scientifici e, di conseguenza, per i termini tecnici'. Ė così possibile trovare osservazioni acute come quella inserita all'interno di una recensione a un Vocabolario toscano della medicina, compilato da Francesco Cionacci ${ }^{8}$ e rimasto manoscritto:

5. «Or io non voglio qui ragionarci degli abusi, e difetti della lingua popolare, che coll'uso, non già a forza dei precetti s'apprende» (Galleria 1697, II: 5).

6. «... più proporzionata all'esercizio delle scienze la nostra lingua molto adatta per altro alla coltura delle buone lettere» (Galleria 1697, II: 5).

7. A percepirne la forte carica innovativa furono proprio gli intellettuali come Fardella che in quel periodo iniziarono a essere chiamati giornalisti. Cosi secondo le ricostruzioni di Folena i «giornalisti sono definiti dapprima, in Italia come in Francia, i Gesuiti compilatori del Giornale di Trévoux: dal 1703 al 1707 il termine sembra restare in questo ambito limitato e specifico, poi il Maffei nel 1710 attribuisce il nome di giornalisti agli autori del Giornale de' letterati d'Italia» (Folena 1983: 18). Da qui in avanti il termine giornalista non sarà più scritto in corsivo.

8. Lo stesso personaggio approvò la pubblicazione del Vocabolario dell'arte del disegno di Filippo Baldinucci; segno che Cionacci fosse sensibile all'ampliamento lessicale della lingua italiana verso cose tecniche. 
Incredibile sarebbe il vantaggio, che da questa sorta di opere ne provverebbe alla nostra lingua, quando ci fossero persone dotte e sperimentate, le quali si prendessero la fatica di compilare lessici e vocabolari particolari di qualunque arte e scienza, e ne spiegassero i termini e le voci: poiché ciò faciliterebbe ad ognuno il modo di scrivere di qualunque cosa e d'intendere gli autori che trattano materie lontane dalla volgar cognizione, e ciò amplierebbe di molto l'insigne Vocabolario degli Accademici della Crusca, il quale in questa parte vien giudicato essere ancora difettuoso. (GLI 1714, XVIII: 446-447)

Il giornalista anonimo, in questo passo ${ }^{9}$, dopo aver elogiato la grande fatica di Cionacci per aver schedato una grande quantità di voci inerenti il campo medico (per cui il recensore la definisce «piuttosto una selva, che un vocabolario finito», GLI 1714, XVIII: 446), esprime totale adesione al dibattito linguistico contemporaneo, considerando fondamentale e vantaggiosa per chiunque la pubblicazione di un'opera lessicografica tecnica. L'osservazione continua poi con un appunto sul vocabolario della Crusca che - nonostante la terza impressione del 1691 avesse accresciuto notevolmente le voci anche, e soprattutto, di natura tecnica - non aveva ancora soddisfatto le aspettative degli scrittori di cose tecniche. Ecco poi l'esortazione finale dell'osservazione nella quale si augura il completamento dell'opera di Cionacci, così da poter aggiungere alla Crusca un vocabolario medico:

Noi vorremmo che qualche valentuomo desse parimente l'ultima mano a questo Vocabolario del Cionacci intorno alla Medicina. Cosi pure sarebbe utilissimo che ne avessimo uno della Matematica, uno della Navigazione, uno della Legge, uno della Mascalcia, e così discorrendo per tutte le Arti e Scienze. In Latino e in Francese ne sono usciti parecchi di questa fatta, i quali la nostra Italia può invidiare alle altre nazioni. (GLI 1714, XVIII: 447-448)

L'intervento si conclude con il riferimento ai lessici tecnici redatti in latino o in francese, scatenando l'invidia e l'imbarazzo della lingua italiana per non avere ancora colmato tali lacune lessicali ${ }^{10}$.

Dopo essere entrati in medias res occorre specificare i presupposti sui quali ebbe inizio il Giornale de' letterati d'Italia ${ }^{11}$. Nella dedica al duca Ferdinando di Toscana viene esposto il progetto intellettuale con la promessa di «racchiudere tutto ciò che di più eccellente negli ultimi anni hanno prodotto, e

9. La porzione di testo citata, così come quella seguente che si citerà dopo, reca a inizio e fine un asterisco: nel GLI gli asterischi indicano, com'è evidenziato a piè di pagina, un'osservazione del giornalista, perciò un contenuto soggettivo.

10. In realtà, un vocabolario della navigazione era già stato pubblicato esattamente un secolo prima in calce a un trattato marinareso: Pantero Pantera, L'armata navale divisa in doi libri nei quali si ragiona del modo che si ha a tenere per formare, ordinare e conservare un'armata maritima. Con molti avvertimenti necessari alla navigatione et alla battaglia. Con un Vocabolario nel quale si dichiarano i nome e le voci marineresche, in Roma, appresso Egidio Spada, 1614; non un vocabolario, ma un'opera di traduzione dal latino fu quella di Giovan Battista De Luca, Il dottor volgare, overo il compendio di tutta la legge [... .] moralizato in lingua italiana per istruzione e comodità maggiore di questa pronuncia, Roma, nella stamperia di Giuseppe Corvo, 1673 nella quale si possono trovare parecchi termini riguardanti il diritto.

11. Per un quadro esaustivo sul GL/ si vedano Castronovo-Ricuperati 1976; De Michelis 1979; Generali 1984; Maffei 2009 : 11-29; Del Tedesco 2012. Preziosi gli indici di Fantato (2012). 
d'ora innanzi produrranno gl'italiani ingegni» (GLI I, 1710:5) 12. La volontà espressa di creare un nuovo giornale che contenesse una nuova linfa arrivò da uno dei primi sostenitori (in seguito divenuto uno dei primi accusatori) della Galleria di Minerva, Apostolo Zeno. Designato come un «traghettatore» (Di Brazzà, 2012: 156), non solo ideologico, Zeno catalizzò attorno a sé e al giornale nuove figure appartenenti alla sfera culturale, convinto che Albrizzi e la sua Galleria fossero eccessivamente piegati alle esigenze dei lettori ${ }^{13}$. II nuovo gruppo di giornalisti, desideroso di far conoscere e apprezzare in Europa la propria rivista, non mancò da subito di accogliere tra le pagine dei primissimi tomi la querelle esplosa qualche anno prima tra Orsi e Bouhours. A riguardo, è bene ricordare che le Considerazioni sopra un libro francese di Orsi destarono immediatamente l'attenzione degli intellettuali europei e venivano difese o attaccate sui giornali dell'epoca. Difese da Muratori che procurò un'importante recensione sugli «Acta Eruditorum» di Lipsia; attaccate, invece, dai gesuiti della rivista «Memoires de Trévoux», che dal canto loro proteggevano l'ormai defunto abate Bouhours ${ }^{14}$.

Tra gli esponenti che tracciarono la politica culturale e che sicuramente contribuirono al progressismo del giornalismo veneto erudito, si deve ricordare Antonio Vallisneri che, oltre a essere medico e scienziato, condusse insieme a Zeno e Maffei una decisa battaglia per il rinnovamento della letteratura e della lingua italiana. Non a caso, nel primo dei tre Supplementi al Giornale de'letterati d'Italia, stampati con cadenza irregolare tra il 1722 e il 1726, Vallisneri ritenne opportuno pubblicare anonimamente uno scritto a sostegno dell'utilizzo della lingua volgare nella composizione di ogni genere di opere. II titolo della dissertazione Che ogni italiano debba scrivere in Lingua purgata Italiana, o Toscana, per debito, per giustizia e per decoro della nostra Italia si rivelò un'appassionata difesa della lingua italiana e non un semplice scritto d'occasione. Può essere utile citare la difesa condotta da Vallisneri, che si sviluppa coscienziosamente su punti già accennati da intellettuali contemporanei, e che si avvale di cinque requisiti sostanziali posseduti dall'italiano:

La prima si è che il nostro Idioma sia ricco e copioso di voci, e di sinonimi, a fine di poterne far libera elezione de' migliori, e de' più confacenti all'eleganza dello stile, e alla sua proprietà del parlare, conforme il soggetto, di cui imprende a ragionare. 2. Che porti seco agevolezza e comodità di favellare [...]. 3. Che sia capace di molte e varie figure, e di forme nobili ed ingegnose, che conferiscono agli artefici dell'eloquenza la varietà dello stile. 4. Che sia di suo-

12. Cf. Gatta (2014: 303-304).

13. In merito si veda Piccioni (1949: 27).

14. Cf. Acta eruditorum (1705, VIII: 370-379) e le obiezioni dei padri gesuiti francesi apparse nel periodico scientifico-letterario Memoires de Trévoux (1705, II: 237-272), (1705, III: 402-436), (1705, IV: 557-574). L'autore dei vari articoli dei Memoires (di cui non si conosce il nome) ne giustifica la divisione in tre parti: «Nous avons crù que l'on verroit avec plaisir un long extrait du Livre de Mr. le Marquis Orsi écrit dans une langue et imprimé dans un païs étranger; d'ailleurs, l'interêt que nous devons prendre à la memoire de l'Auteur qu'il attaque, ne nous a pas permis de rapporter les Objections de l'Ecrivain Italien sans y répondre, ce qui nous oblige à partager en trois cet extrait» (Journal de Trévoux, II: 272). È opera di Muratori aver raccolto un'edizione delle Conversazioni (1735) nella quale confluirono, oltre a una biografia dell'Orsi, tutti gli scritti intorno alla polemica italo-francese. Sulla querelle Orsi-Bouhours si vedano almeno Gensini (1993: 51-97), Matarrese (1993), Madonia (1998: 77-89), Viola (2012). 
no dolce e spedito nella pronunzia. 5. Che finalmente tutto ciò si intenda tanto della prosa, quanto del verso. (GLIsup 1722: 267-268)

Sempre nello stesso articolo di denuncia, l'autore si lamenta dell'incapacità di chi, uscendo da un'istruzione scolastica, non riesce a raggiungere un grado di scrittura elementare: "Quanti solecismi barbarismi, maniere di dire abbiette, vocaboli barbari, oscuri, plebei, continuamente senza nausea si leggono? Quale stile duro, aspro, confuso, disaggradevole si ritrova? Quali, e quanti errori nella sola ortografia, e nelle più trite regole della gramatica s'incontrano?» (GLIsup 1722: 269-270). L'accusa si rivolge, come ben si può vedere, anche agli usi lessicali, ritenuti inadeguati e il più delle volte oscuri; l'addizione di un vocabolario povero a un'ortografia scorretta rende, per Vallisneri, la prosa aspra e confusa. Dalle parole dello scienziato si può ricavare una critica anche a coloro che, per difendere interessi personali, proponevano lo studio del latino a discapito dell'italiano. Un esempio riportato nella lettera ha come oggetto l'ignoranza dei medici camuffata dalla lingua usata per le cure, e cioè da parole greco-latine o addirittura arabe, incomprensibili dal popolo. Avvicinandosi anch'esso come Fardella a un pensiero progressista, Vallisneri sostiene che l'italiano del suo tempo è più evoluto, e quindi più perfetto, di quello delle origini, poiché la lingua è frutto di una lenta e continua trasformazione. Da questi pochi pensieri espressi nel GLI, emerge un incoraggiamento dell'autore verso una ricerca lessicografica, al fine di poter aumentare le capacità espressive dell'italiano ${ }^{15}$. Questo deciso interesse nei confronti della lessicografia, come abbiamo visto, sembra essere il filo conduttore del pensiero linguistico che dalla Galleria di Minerva si protrae fino al GLI.

Avevamo accennato a una recensione su un incompiuto e manoscritto Vocabolario medico, e si era riportato il monito del recensore per un intellettuale futuro che completasse l'opera, poiché necessaria all'idioma italiano. Ad aver trasformato il consiglio in opera fu proprio Vallisneri, che col suo Saggio d'istoria medica e naturale - rimasto anch'esso incompiuto - contribuì all'ampliamento lessicografico. Nell'ultimo volume del GL/ del 1740, all'interno di un articolo celebrativo su Antonio Vallisneri, il giornalista anonimo, ricordando il Saggio d'istoria medica, scrive:

il suo disegno [di Vallisneri] è differente da quello degli Accademici della Crusca; essi apportano voci, che sieno usate da Autori Classici di lingua, il Vallisneri spiega le idee che i Medici e Storici naturali unirono sotto di esse, nulla importando a lui da chi dette; e sì nel parlare di quel riguardevole corpo della Crusca, che della lingua Italiana il più bel fior ne coglie, come nel giudicio di questa sua fatica, egli è pieno al suo solito di modestia, e pronto a correggersi e rimettersi alle chiare ragioni di chiunque gli dimostrasse gli errori suoi. Le voci da lui spiegate sono al numero di ottocento e più. (GLI XL, 1740: 136)

Con queste parole conferma l'impegno culturale e linguistico del defunto scienziato che aveva portato avanti la stesura del Saggio per donare alla lingua italiana la conoscenza dei vocaboli settoriali. Impegno che fu, come si nota dalle poche righe del recensore, lodato per lungo tempo.

15. Si veda Vallisneri 2013: 22. 
Sotto l'ala del GLI, oltre alla Repubblica delle Lettere, trovò riparo anche la Repubblica dei Matematici, decisi, secondo il disegno dei fondatori, a usare il volgare anche nella cultura scientifica. Che sia un passo di notevole importanza lo si capisce sia dalle recensioni, sia dagli articoli che traducevano in italiano opere filosofiche e matematiche, tramite le quali vi era un notevole incremento lessicale ${ }^{16}$. Si può affermare, perciò, che l'apertura alla modernità fosse la cifra del GLI, che ospitò dalle semplici recensioni della grammatica del Buommattei (1722), della quarta edizione del Vocabolario della Crusca (1727), del De vulgari eloquentia volgarizzato dal Trissino (1710), ai semplici appunti bibliografici come nel caso delle Osservazioni per lo scrivere italiano e latino e degli Elementi della lingua toscana (1723), per arrivare, come abbiamo visto, a interventi e osservazioni mirate su questioni di lingua. Per ragioni di economia darò solo notizia di alcuni scritti di natura puramente linguistica: si avrà perciò il giornalista che si interroga sull'origine della lettera $<k>>^{17}$, vagliando le varie teorie e partendo dalla lingua fenicia; un altro articolo si sofferma sulla natura della parola mancia.

Per chiudere il capitolo sul GLI guarderò un altro articolo apparso nel 1711 dal titolo Controversie gramaticali intorno alla lingua italiana. Si riporta qui l'introduzione:

Le lingue viventi hanno dato e daranno di continuo un ampio soggetto alle contese letterarie, tanto in riguardo alla pronunzia e alla scrittura, quanto in ordine alle voci, alle maniere di dire, e a tutto ciò che gramatica generalmente si appella. La nostra volgar lingua, e forse più ch'altra mai, è stata sempre sottoposta a sì fatti dibattimenti; e comechè i molti libri di tante persone versatissime in questa materia, usciti in diversi tempi e occasioni, dovrebbono aver posto fine a i contrasti, e dato a conoscere qual parte a seguitare, e quale a sfuggir si abbia, gli animi non per tanto non si sono acquetati, anzi pare che i medesimi maestri non ben d'accordo fra loro nel fissare sotto regole certe e sotto comuni principi la ortografia e la pronunzia, abbiano più tosto giustificate, e tenute in piedi, che decise e sopite le differenze [...]. Innanzi però di tutto premetteremo alcune regole generali, le quali possono esser giovevolissime per ben giudicare in tali materie. (GLI 1711, t.8: 120-121)

Il giornalista anonimo, dopo aver condotto un breve excursus sulla vexata quaestio linguistica, elenca di fatto le principali regole che ritiene aver percepito concordi tra tutti gli autori. La prima riguarda le voci che prima di tutte andrebbero usate, cioè quelle già consolidate e comprovate dai buoni scrittori e già in uso da tempo; la seconda e la terza mettono in guardia dalle parole definite «alla giornata» e dai modi di dire presi in prestito dal latino o dal francese; interessante il quinto punto sull'ortografia da non ricercare nelle scritture degli antichi, ma dai libri moderni; si sottolinea poi l'uso di alcune parole che possono essere scritte in diversi modi (comunque giusti); ultimo appunto sulla pronunzia, molto libera e differente nelle varie parti d'Italia.

16. Termini come differenziale, ascisse, isocrona, forze tangenziali ecc., tradotti da scritti di Newton, Leibniz, Huygens, vedevano la luce per la prima volta nel GLI (cf. Roero 2012: 73).

17. Cf. Benvoglieni (1726, GLISup III: 217-248). 


\section{La continuazione ideale del GLI}

Se fin qui abbiamo visto la formazione di una ideologia linguistica, all'interno del GLI, continuatrice del pensiero seicentesco di marca galileiana e rediana, adesso si farà riferimento a una meteora giornalistica: le Osservazioni letterarie.

La rivista, pubblicata tra il 1737 e il 1740 in sei volumi, fu fondata da Scipione Maffei, già collaboratore del GLI, e sembra tornare sui passi preilluministici che distinguevano l'Introduzione del Giornale di parecchi anni prima. Pochissimi sono i riferimenti alla lingua italiana, più che altro legati al suo uso nell'opera teatrale ${ }^{18} \mathrm{O}$ in poesia. Parlando del confronto poetico tra varie lingue, Maffei scrive ad esempio:

Singolarmente credo gioverebbe il far uso frequente delle trasposizioni. Che diverrebbero i versi di Virgilio e d'Omero, se tessuti fossero con la natural costruzione e con quella giacitura di parole, secondo cui si parla ordinariamente? Delle moderne lingue alcune così procedon sempre, e non possono alterare in verun modo cotal testura. Altre hanno più trasposizioni ordinarie e fisse dalle quali non si possono dipartir mai. L'italiana all'incontro e può trasporre, e non trasporre; e parlar naturalmente quando fa al caso, e allontanarsi dall'ordine famigliare e comune delle parole quando torna bene. (OL 1737, I: 320-321)

L'intervento di Maffei, che è da ascriversi nel più ampio dibattito settecentesco riguardante l'ordine naturale della lingua ${ }^{19}$, ritrae il pensiero di chi nel Settecento difendeva la lingua italiana e il suo ordine delle parole, caratterizzato dalla non troppa rigidità delle strutture. II riferimento, come si legge, non va solo alla poesia, ma anche alla lingua propriamente parlata, poiché anche se un membro frastico viene spostato dalla sua naturale posizione si capirà lo stesso il senso della frase.

Procedendo in altra direzione, ci spostiamo adesso su una recensione apparsa sempre nel primo tomo a un volume di Prose e poesie dell'abate Gerolamo Tagliazucchi. Riporto interamente l'incipit:

Questo degno Soggetto, ch'è professor d'eloquenza nella Regia Università di Torino, dopo elegante e veridica dedicatoria all'Alt. Reale di Vittorio Amedeo Duca di Savoja, dà qui una raccolta di componimenti, dalla quale si può arguire l'ottima strada, per cui guida quella gioventù nella carriera delle belle lettere. Dibatte prima se nelle Scuole oltre alla latina debbasi introdurre anche lo studio della volgar lingua. Con declamazion latina mostra di sostenere la negativa; poi con Orazion volgare fa dottamente conoscere come a gran torto nelle Università

18. Per i riferimenti agli interessi musicali di Maffei cf. Folena (1983: 235-261). Più in generale, sulle Osservazioni letterarie cf. Piccioni (1949: 91-92), in cui è presente una breve antologia di alcuni estratti alle p. 94-116. Maffei era personaggio centrale nella discussione sulla traduzione e così anche su problemi linguistici.

19. Il dibattito sull'ordine logico naturale prende il via con la polemica Orsi-Bouhors e continua per tutto il Settecento nella discussione sul genio delle lingue. A tal proposito cf. Schwarze (2006: 167-182), Tomasin (2011: 72), Serianni (2012: 108-110), Marazzini (2016: 638-639). 
e nelle Scuole si trascura d'insegnare e di coltivare anche la lingua Italiana; necessaria essendo l'una e l'altra, e non potendosi senza qualche applicazione, e senza qualche studio di gramatica, e sopra tutto senza lettura de' buoni Autori, saper mai né pur la nostra: talché chi si crede, per esser nato in Italia, di naturalmente saperla, si resta come gl'idioti plebei in una vergognosa ignoranza della propria favella. (OL 1737, I: 256-257)

Si può ben comprendere la vicinanza ideologica nei confronti dello studio della lingua italiana a scuola, ritenuta indispensabile per conoscere in modo appropriato la propria lingua materna costruita su solide basi grammaticali percepibili solo attraverso l'esercizio. Più avanti, Scipione Maffei continua la recensione con brevi cenni sulla grafia di alcune parole presenti nell'opera:

Alquanto più è dispiaciuto a molti, di osservarci quella novità d'ortografia, io ò, quello à, quelli an, overo anno: ma forse è da attribuire allo stampatore, o al correttor della stampa. Nulla è più importante e più necessario per promuovere una lingua e per renderla accetta e agevole a gli stranieri, del fissarsi una volta, ed accordarsi per quanto è possibile gli Scrittori tutti, almeno in ciò che spetta a certi punti più frequenti e più comuni, nell'istessa maniera di scrivere. Ora e' sarebbe a ciò troppo ripugnante e contrario, l'abbracciar bizarrie e novità non mai praticate nel 1300 e nel 1500, che sono i due secoli d'oro per la nostra lingua; né accettate in veruna delle quattro edizioni del Vocabolario della Crusca, l'una delle quali si fa attualmente; né approvate dal comune di que' Letterati che oggidì in Italia per via di stampe più si distinguono (OL 1737. I: 258-259).

Proposizioni come questa si muovono in perfetta sintonia e confermano le altre, scritte anni prima per il GLI. Basta riprendere pochi luoghi per «cogliere un sentore di quella corrente prepuristica, di quell'atticismo arcaizzante che metterà radici soprattutto nei confini dello stato veneto» (Folena 1983: 26):

Vedrebbero allora che quel buon senso ch'essi con tanta carità ci vanno augurando, nacque fra noi al nascere di nostra lingua, e già nel secolo del 1300 a perfezione era giunto; vedrebbero ch'egli non mancò in Italia giammai, benchè nel XV secolo alquanto meno si coltivasse, e benchè nel XVII in alcuna provincia patisse disastro; e vedrebbero finalmente, ch'egli fiorisce ancora oggi giorno quanto in altro tempo mai fosse (GLI 1710. I, Introduzione: 26).

Al di là delle considerazioni sul gusto di una lingua cristallizzata, è necessario considerare l'apertura di Maffei nei confronti delle altre lingue. Stando alle parole del veronese, inoltre, si dovrebbe in parte riconsiderare la salda convinzione dell'italiano come unica lingua degna di tradurre il latino e il greco: è proprio nelle OL che l'autore elogia, ad esempio, l'inglese capace di tradurre con una parola ciò che in italiano si tradurrebbe con due. II grado di tollerabilità, seppur basso, verso le altre nazioni, comunque, echeggiava in alcune righe scritte a proposito dell'arricchimento che proverrebbe dal dialogo tra due lingue: 
Così dee fare chi è costumato e chi è dotto. Giova l'emulazione anche fra le nazioni, quando serve a eccitarsi scambievolmente alle belle imprese. Ma né questa dee mai pregiudicare alla civiltà, né alla stima vicendevole. Non bisogna parimente che la passione per la propria nazione acciechi mai e impedisca l'intender ragione, né faccia credere che si disprezzi un Autore, quando qualche cosa si riprende in esso. Non c'è Italiano di conto, che non faccia molta stima de' Francesi, né Francese di voglia, che non faccia molta stima degl'Italiani (OL 1737, I: 266).

Frasi di tal genere sono rare a trovarsi in quegli anni, e lo stesso Maffei annota queste righe all'interno di una recensione e subito dopo aver elogiato l'autore del libro Paragone della Poesia tragica d'Italia con quella di Francia per non esser caduto nella «parzialità verso l'una o l'altra delle due nazioni» (OL 1737, I: 236). Ci si trova perciò con un pensiero assai moderno e comunque legato al pensiero classico dell'emulatio. Oltre al paragone tra modelli diversi, si avvertirà un arricchimento - specialmente lessicale - dalle traduzioni dal latino o dal greco; perché chi scrive sforza maggiormente la propria retorica al fine di trovare le parole più adatte.

\section{Tra antico e moderno: Lami, Gozzi, Baretti}

Accenneremo, infine e brevemente, ad altre quattro riviste: le Novelle Letterarie di Giovanni Lami, la Gazzetta Veneta, l'Osservatore Veneto di Gaspare Gozzi (l'ultimo titolo impregnato di un profumo europeo, tanto da ricordare lo Spectator dell'Addison): e la Frusta letteraria di Giuseppe Baretti.

Nel florido periodo di pubblicazione delle Novelle (1740-1792), improntate su un nuovo schema di novità bibliografiche, e che seguiva realmente le orme dei dibattiti di ciò che era avvenuto già da anni ormai in Francia e Germania, il Lami - che fu a capo della direzione fino al 1769 - apportò un respiro enciclopedico al giornalismo veneto erudito ${ }^{20}$. Citeremo solo un brevissimo, ma esaustivo intervento del Lami riguardo un'iniziale ideologia sulla lingua italiana di metà Settecento. Si tratta di un'avvertenza che annuncia la nascita di un nuovo giornale:

Cominciasi qui l'anno prossimo un nuovo Giornale d'Italia; giacché i paesi di qua da i monti non erano più informati delle opere, che nell'Italia continuamente si stampano, da che finì la Biblioteca Italica di Ginevra. È vero che i Giornalisti di Lipsia e di Trevoux vanno riferendo di tempo in tempo alcuni de' libri italiani, ma facendo questo ora con molta passione, ora con molta negligenza, hanno piuttosto dato un impulso alla determinazione che si è qui fatta. Questo Giornale sarà sul piede di quello del Sig. Apostolo Zeno, che stampavasi in Venezia, colla sola differenza che questo sarà, come si è detto, in Franzese, giacché la lingua Italiana non è in grande uso fuori d'Italia (NL 1740, t.1: 784)

20. Per un approfondimento sulle NL si vedano Capecchi (2008: 55-80); Nicoletti (2008: 3-20); Castori (2011: 65-76). 
Ritengo che un avvertimento bibliografico come quello appena citato spicchi notevolmente su molti altri esempi cui avrei potuto attingere, perché in esso si discute sul grado d'uso della lingua italiana all'estero. Non che sia un dato innovativo, quello del Lami; ma in quegli anni, nei quali imperava la difesa del primato dell'italiano sulle altre lingue europee - soprattutto in ambito traduttologico pochi avrebbero resa pubblica una tale affermazione. Inoltre, nella notizia si fa riferimento all'intento dei giornalisti esteri (ovviamente, ci si riferisce agli Acta eruditorum e ai Memoires) di inserire nelle stampe le più recenti pubblicazioni italiane, con vani risultati però.

Sul versante nazionale Lami manifesta il culto della toscanità: così emerge dalla definizione che dà di lingua comune che insieme alla «lingua Toscana, sono sinonimi, come egregiamente pruova il Cavaliere Leonardo Salviati ne' suoi Avvertimenti e come ha sempre confermato l'uso de' buoni Scrittori» (NL 1740, t.1: 804). Siamo di fronte a concetti in cui i giornalisti esprimono considerazioni prepuriste, che si ritrovano anche nelle pagine delle NL e della Gazzetta Veneta del Gozzi, sulla quale ci soffermeremo tra non molto. Continuando la disamina delle NL, ci s'imbatte in un problema che ha attraversato, come finora abbiamo visto, il pensiero di tutti gli intellettuali giornalisti: la carenza di un adeguato vocabolario scientifico. Lo stesso recensore lamenta l'abbandono di un progetto assai ambizioso di traduzione, per via delle numerose difficoltà:

Dopo ch'io ebbi formato il disegno di stampare, tradotto in lingua italiana, il celebre Dizionario Inglese delle Arti e delle Scienze di Chambers, e pubblicatane la Prefazione per saggio, alcuni accidenti non previsti, ma sopra tutto le grandi difficoltà che dovettero superarsi per condurre a fine un buono ed intero Nomenclatore Italiano, che dovea servir di filo alla lunga opera della Traduzione, m’hanno fatto differire l'esecuzione del mio Progetto. (NL 1747, t.8: 746)

E a proposito di lessicografia, un decennio dopo il Lami, nel primo tomo della nuova impressione delle NL del 1770, pubblica una recensione alla edizione aggiornata dei Synonymes François a cura degli abati Girard e Bauzèe. Come ormai norma confermata, il giornalista riporta le osservazioni non solo in merito all'opera da recensire, ma anche sulla situazione lacunosa della lessicografia italiana:

Quest'opera mortifica alcun poco la vanità di noi altri Toscani, che ci vantiamo di essere i primi ed i più esatti a dar regola nelle lingue vive, ed a fabbricare la Grammatica nell'idioma Italiano. Ė già molto tempo che negli altri Paesi si va fissando con estrema precisione il significato vero delle voci del proprio di ciascuno, e si va dimostrando non esservene di quelle che possano chiamarsi Sinonime, e tuttavia stiamo indolenti a veder sussistere il pregiudizio per il nostro volgare. (NL 1770, I: 782)

Un commento che aderisce bene al precedente scritto di esattamente vent'anni prima: un arco cronologico nel quale, evidentemente, ancora si sentiva la necessità di implementare il repertorio lessicale della lingua italiana. Non sorprende tanto la critica, rivolta soprattutto agli accademici (di 
cui il Lami fece parte) $)^{21}$, quanto l'aperta dichiarazione di prendere esempio dagl'intellettuali europei - nel nostro caso, francesi - affinché un volume simile potesse essere stampato anche in Italia.

Sono anni in cui il duro conservatorismo intellettuale s'incontra con le idee antilluministiche, e in parte progressiste, di Gozzi. Nella sua lunga esperienza giornalistica (ma non solo) ${ }^{22}$, Gozzi interviene più volte su alcune questioni linguistiche, specie riguardo al tipo di educazione da adottare nelle scuole nei confronti dell'italiano. Dalla Gazzetta Veneta, passando per l'Osservatore veneto e, infine, approdando al Sognatore italiano, Gozzi adotta un programma dichiaratamente in contrasto con le teorie dell'illuminismo e del razionalismo che pervadeva la cultura veneta del periodo. L'aperta battaglia per una giusta educazione linguistica rispecchia, a tal proposito, la spinta progressista e l'interesse pedagogico dell'erudito veneto ${ }^{23}$ che, al di là del rifiuto della tradizione, sente comunque l'esigenza di specificare l'utilità dell'insegnamento della grammatica e della lingua latina che potrà servire agli «Ecclesiastici, Legali, e Medici» (Gozzi, 1836: 10).

Accennerò, per tirare le fila e far parlare Gozzi, a un articolo apparso nell'agosto del 1761 sull'Osservatore Veneto, nel quale l'autore affronta il problema della brevità e della stringatezza dello stile. Dopo aver ricordato i modi concisi dello scrivere di Sallustio e Tacito apprezzando di più l'autore degli Annali, Gozzi consiglia che per scrivere bene siano necessari due fattori: la conoscenza della materia trattata e il possesso della lingua in cui si scrive «accioché ogni pensiero si presenti con adatti vocaboli, per non abbisognare di lunghi giri a spiegarsi» (Osservatore, 29 agosto 1761).

In fatto di stile, infine, si esprime anche Giuseppe Baretti, fondatore della Frusta letteraria (17631765) e animatore di tutti gli interventi firmati con lo pseudonimo di Aristarco Scannabue. In un contributo venuto alle stampe nel novembre 1763 (Frusta IV, I) Baretti pone a confronto due tipi di stile: quello di Bevenuto Cellini «uomo ignorantissimo», con quello dell'abate Antonio Genovesi, considerato uomo dotto. «Voi troverete che quello del Cellini è semplice, chiaro, veloce, e animatissimo, e quello del Genovesi intralciato, languido, stiracchiato e scuro. E perché questo?» (Frusta IV, I: 45). E continua con una teoria che vede nella natura il fattore determinante per uno stile buono e semplice che è stato concesso a Cellini e che lo ha spinto a «mettere il Nominativo innanzi al Verbo, e dietro al Verbo l'Accusativo, o qualunque altro caso gli occorreva per rendere il suo Discorso grammaticale e secondo l'indole del Parlar fiorentino» (Frusta IV, I: 46). Baretti esprime, in accordo con alcune teorie già formulate da intellettuali già citati prima, quali Gozzi, un'idea linguistica fondata sull'uso di un

21. «Un Accademico della Crusca vi lesse mesi addietro una Lezione, nella quale si accinse a provare questa verità, ed a persuadere quanto necessario fosse che noi analizzassimo il nostro linguaggio per scoprire la natural proprietà rigorosa delle parole che adopriamo. Fu lodato il suo impegno, ma chi sa quanto staremo ad avere un libro simile a quello che annunziamo?» (NL 1770, I: 783). Sui rapporti tra Lami e la Crusca si veda il saggio di Giudici (1969: 263-287), Vitale (1986: 349-382), Nicoletti (2002: 1-34).

22. Le questioni linguistiche di Gozzi si possono ben leggere nelle lettere e in altre opere, oltre ai giornali.

23. A proposito dell'interesse verso la pedagogia di Gozzi è opportuno segnalare Rosada (1989: 79-93). Sulle posizioni progressiste e le spinte antilluministe Cataudella (1989: 445-453) e, in parte, Spezzani (1989: 95-108). 
periodo semplice, senza l'esigenza di imitare gli auctores trecenteschi (riferendosi a Boccaccio e al Firenzuola), senza inserire verbi in clausola se non strettamente necessari.

\section{Conclusioni}

La testimonianza emersa dall'analisi di alcuni articoli tratti dalle riviste nate nella prima metà del Settecento pare legittimare alcune considerazioni conclusive. Avvalendoci delle autorevoli parole di Folena e Schiaffini, nella premessa avevamo parlato di un'ideologia linguistica non più - e non solo - legata alla crisi, ma in rotta verso un proprio rinnovamento. Posto ciò, si è potuto affrontare il dibattito linguistico settecentesco da un altro punto di vista: la volontà dell'italiano di emulare le altre lingue. Un'emulazione certo che già gli intellettuali giornalisti avevano ben compreso essere preziosa e che riflette soprattutto un'opinione comune, che va dai primi numeri della Galleria agli ultimi delle Novelle letterarie.

L'analisi ha perciò permesso di osservare non solo il pensiero linguistico dei più grandi nomi (ricordiamo Zeno, Vallisneri, Gozzi, Baretti, Lami), ma una concreta tendenza ideologica delle riviste alle quali gli intellettuali erano soliti collaborare con saggi di interesse etnolinguistico ${ }^{24}$ o con recensioni. E proprio dalle ultime si è potuta evidenziare l'apertura linguistica dei giornalisti25, che proposero l'imitazione degli sforzi dei lessicografi o grammatici stranieri o che elogiarono le poche imprese atte alla creazione dei linguaggi scientifici. E se di idea progressista della lingua si sta parlando, bisogna tener conto anche del secondo aspetto di sicuro interesse: l'attenzione verso lo studio della lingua italiana. L'azione educatrice promossa dai giornali caldeggiava lo studio dell'italiano a discapito del latino, percepito come artefatto e non utile alla comprensione quotidiana.

Anche quando a essere chiamata in causa è la Crusca col suo vocabolario, i recensori (pur del calibro di Lami, membro dell'Accademia della Crusca) consigliano di non cristallizzare la lingua attraverso la schedatura di voci tratte da autori trecenteschi, ma di ampliare la sua conoscenza attraverso nuovi repertori lessicografici, come ad esempio i vocabolari scientifici. Sembra quindi che nei giornali presi in esame esista una linea programmatica comune, volta a creare un nuovo terreno su cui i futuri intellettuali avrebbero potuto camminare e plasmare una nuova identità linguistica.

24. Un esempio è l'articolo sull'origine degli Etruschi da parte di Maffei sulle OL, cf. Maffei (1739, OL IV: 5-41).

25. È utile ribadire ancora una volta che il termine giornalista non va inteso in senso moderno. Ad assolvere le funzioni di giornalisti erano letterati, eruditi, scienziati e intellettuali. 


\section{Bibliografia}

Albrizzi, Girolamo (1696), «A' letterati d’Europa», Galleria di Minerva, I, Venezia, Presso Girolamo Albrizzi.

Benvoglieni, Uberto (1726), «Alcune osservazioni intorno all'origine del K appresso degl'Italiani, di Tuberone Guntolibei, Cittadino Massetano», Supplementi al Giornale de'letterati d'Italia, III, Venezia, Appresso Gio. Gabriello Hertz, p. 217-248.

Brunetti, Francesca (ed.) (2009), Introduzione, in Scipione Maffei, Letterati d'Italia: introduzione al Giornale (1710), Venezia, Marsilio.

Capecchi, Silvia (2008), "Lumi e letteratura nella seconda serie delle "Novelle letterarie"», in Silvia Capecchi (ed.), Giornali del Settecento fra Granducato e Legazioni. Atti del convegno di studi (Firenze, 17-19 maggio 2006), Roma, Edizioni di Storia e Letteratura, p. 55-80.

Castori, Claudia (2011), «Le "Novelle letterarie" nei giornali veneziani del Settecento», in Alberto Zava, Ilaria Crotti, Enza Del Tedesco, Ricciarda Ricorda (ed.), Autori, lettori e mercato nella modernità letteraria, Pisa, ETS, p. 65-76.

Castronovo, Valerio, Capra, Carlo e Ricuperati, Giuseppe (1976), La stampa italiana dal Cinquecento all'Ottocento, Roma-Bari, Laterza.

Cataudella, Michele (1986), «Antilluminismo e progresso nell'ultimo Gozzi», in Ilaria Crotti e Ricciarda Ricordi, Gasparo Gozzi. Il lavoro di un intellettuale nel Settecento veneziano. Atti del convegno (Venezia-Pordenone 4-6 dicembre 1986), Padova, Antenore, p. 445-453.

Coletti, Vittorio (2000), Storia dell'italiano letterario, Torino, Einaudi.

Considerazioni = Ludovico Antonio Muratori (ed.) (1705), Considerazioni del marchese Giovan Giofesso Orsi bolognese sopra la Maniera di ben pensare ne' componimenti, già pubblicata dal padre Domenico Bouhours della Compagnia di Gesù. S'aggiungono tutte le scritture che in occasione di questa letteraria contesa uscirono a favore e contro al detto marchese Orsi, colla di lui Vita e colle sue Rime in fine, Modena, Bartolomeo Soliani, 2 vol.

De Michelis, Cesare (1979), Letterati e lettori nel Settecento veneziano, Firenze, Olschki.

Del Tedesco, Enza (ed.) (2012), Il Giornale de' letterati d'Italia trecento anni dopo. Scienza, storia, arte, identità (1710-2010). Atti del convegno, Padova, Venezia, Verona, 17-19 novembre 2010, Pisa-Roma, Fabrizio Serra.

Di Brazzà, Fabiana (2012), «Apostolo Zeno dalla "Galleria di Minerva" al "Giornale de' Letterati”», in Enza del Tedesco (ed.), I/ «Giornale de' letterati d'Italia» trecento anni dopo. Scienza storia, arte, identità (1710-2010). Atti del convegno, Padova, Venezia, Verona 17-19 novembre 2010, Pisa-Roma, Fabrizio Serra.

Fantato, Michela (2012), Indici del "Giornale de' letterati d'Italia", Pisa-Roma, Fabrizio Serra. 
Fardella, Michelangelo (1697), «Lettera del sig. abbate Michel Angelo Fardella Prof. d'Astronomia e Meteore nello studio di Padova al sig. N.N. in cui si dimostra quanto sia presentemente corrotta, ed allontanata dal suo primo istituto l'arte di parlare», Galleria di Minerva, Venezia, 1697, p. 1-16.

Folena, Gianfranco (1983), L'italiano in Europa. Esperienze linguistiche del Settecento, Torino, Einaudi.

Fubini, Mario (1954), Dal Muratori al Baretti: studi sulla critica e sulla cultura del Settecento, Bari, Laterza.

Gatta, Francesca (2014), «Giornalismo», in Giuseppe Antonelli, Matteo Motolese, Lorenzo Tomasin (ed.), Storia dell'italiano scritto III. Italiano dell'uso, Roma, Carocci, p. 293-347.

Generali, Dario (1984), «ll Giornale de' letterati d'Italia e la cultura veneta del primo Settecento», Rivista di storia della filosofia, n² 2, p. 243-281.

Generali, Dario (1991), «Introduzione», in Antonio Vallisneri, Epistolario (1679-1710), vol. 1, p. 36-40.

Gensini, Stefano (1993), Volgar favella. Percorsi del pensiero linguistico italiano da Robortello a Manzoni, Firenze, La Nuova Italia.

Giudici, Paolo (1969), «Giovanni Lami e la Crusca», in I romanzi di Antonio Fogazzaro e altri saggi, p. 273-287.

Madonia, Francesco Paolo (1998), «Osservazioni in margine alla polemica Orsi-Bouhours», Esperienze letterarie, 23, p. 77-89.

Maffei, Scipione (1739), «Della nazione etrusca e degl'Itali primitivi. Trattato in quattro libri diviso», Osservazioni letterarie, IV, p. 5-42.

Marazzini, Claudio (2016), «Questioni linguistiche e politiche per la lingua», in Sergio Lubello (ed.), Manuale di linguistica italiana, Berlin/Boston, De Gruyter, p. 633-654.

Matarrese, Tina (1993), Il Settecento, Bologna, Il Mulino.

Nicoletti, Giuseppe (2002), «Orientamenti di poetica e frequentazioni di letteratura contemporanea nelle "Novelle letterarie" di Giovanni Lami (1740-1769)», Studi italiani, n’ 1-2, p. 1-34.

Nicoletti, Giuseppe (2008), «Erudizione e letteratura nei periodici toscani del Settecento», in Silvia Capecchi (ed.), Giornali del Settecento fra Granducato e Legazioni. Atti del convegno di studi (Firenze, 17-19 maggio 2006), Roma, Edizioni di Storia e Letteratura, p. 3-20.

Pennisi, Antonio (1985), «Grammatici, metafisici, mercatanti: riflessioni linguistiche nel Settecento meridionale», in Lia Formigari (ed.), Teorie e pratiche linguistiche nell'Italia del Settecento, Bologna, Il Mulino, p. 83-107.

Piccioni, Luigi (1949), Giornalismo letterario del Settecento, Torino, Utet.

Ricorda, Ricciarda (1989), "Gasparo Gozzi e il giornalismo: la "Gazzetta Veneta", in Ilaria Crotti e Ricciarda Ricorda (ed.), Gasparo Gozzi. Il lavoro di un intellettuale nel Settecento veneziano. Atti del convegno (Venezia-Pordenone 4-6 dicembre 1986), Padova, Antenore, p. 147-165. 
Roero, Silvia (2012), Il Giornale de' letterati d'Italia e la "Repubblica dei matematici", in /l Giornale de' letterati d'Italia trecento anni dopo, p. 61-82.

Rosalda, Bruno (1989), "Gasparo Gozzi tra morale e pedagogia», in Ilaria Crotti e Ricciarda Ricordi, Gasparo Gozzi. Il lavoro di un intellettuale nel Settecento veneziano. Atti del convegno (Venezia-Pordenone 4-6 dicembre 1986), Padova, Antenore, p. 79-93.

Schiaffini, Alfredo (1950), Momenti della storia della lingua italiana, Bari, Leonardo da Vinci.

Schiaffini, Alfredo (1975), «Aspetti della crisi linguistica italiana del Settecento», in Tullio De Mauro e Paolo Mazzantini (ed.), Italiano antico e moderno, Milano-Napoli, Ricciardi, p. 129-165.

Schwarze, Sabine (2006), «ll genio della lingua nella teoria settecentesca della traduzione», in Coluccia, Giuseppe/Stasi, Beatrice (ed.), Traduzioni letterarie e rinnovamento del gusto: dal Neoclassicismo al primo Romanticismo, vol. 2, Lecce, Mario Congedo Editore, p. 167-182.

Scotti Morgana, Silvia (1983), Esordi della lessicografia scientifica italiana. Il «Saggio alfabetico d'Istoria medica e naturale di Antonio Vallisneri», Firenze.

Serianni, Luca (1998), «La lingua italiana dal cosmpolitismo alla coscienza nazionale», in Enrico Malato (ed.), Storia della letteratura italiana, VI, Roma, Salerno.

Serianni, Luca (2012), Italiano in prosa, Firenze, Franco Cesati Editore.

Spezzani, Pietro (1989), "Gasparo Gozzi e la questione della lingua nel Settecento», in Ilaria crotti e Ricciarda Ricorda (ed.), Gasparo Gozzi. Il lavoro di un intellettuale nel Settecento veneziano. Atti del convegno (Venezia-Pordenone 4-6 dicembre 1986), Padova, Antenore, p. 95-108.

Tomasin, Lorenzo (2011), Italiano. Storia di una parola, Roma, Carocci.

Vallisneri, Antonio (1733), «Saggi d'istoria medica e naturale», in Antonio Vallisneri (ed.), Opere fisico-mediche stampate e manoscritte del kavalier Antonio Vallisneri raccolte da Antonio suo figliolo, vol. 3, Appresso Sebastiano Coleti, Venezia, ora in Massimo Rinaldi (ed.), Saggio d'istoria medica e naturale, colla spiegazione de' nomi, alla medesima spettanti, posti per alfabeto, Firenze, Olschki, 2012.

Vallisneri, Antonio (2013), Che ogni italiano debba scrivere in lingua purgata italiana, Dario Generali (ed.), Firenze, Olschki.

Viola, Corrado (2001), Tradizioni letterarie a confronto: Italia e Francia nella polemica Orsi-Bouhours, Verona, Fiorini.

Vitale, Maurizio (1986), «La IV edizione del "Vocabolario della Crusca”. Toscanismo, classicismo, filologismo nella cultura linguistica fiorentina del primo Settecento», in L'oro della lingua. Contributi per una storia del tradizionalismo e del purismo italiano, Milano-Napoli, Ricciardi, p. 349-382. [1 $1^{\circ}$ ed., 1971] 